\title{
Jean-Marie Funel. Le projet, le territoire et autres contingences Regards sur l'action collective
}

\section{Marielle Berriet-Solliec}

\section{(2) OpenEdition}

1 Journals

Édition électronique

URL : http://journals.openedition.org/economierurale/7084

DOI : 10.4000/economierurale.7084

ISSN : 2105-2581

Éditeur

Société Française d'Économie Rurale (SFER)

\section{Édition imprimée}

Date de publication : 30 septembre 2019

Pagination : 119-121

ISSN : 0013-0559

\section{Référence électronique}

Marielle Berriet-Solliec, « Jean-Marie Funel. Le projet, le territoire et autres contingences Regards sur l'action collective », Économie rurale [En ligne], 369 | Juillet-septembre, mis en ligne le 30 septembre 2019, consulté le 07 janvier 2021. URL : http://journals.openedition.org/economierurale/7084 ; DOI : https://doi.org/10.4000/economierurale.7084 
Jean-Marie FUNEL, 2018

\section{Le projet, le territoire et autres contingences Regards sur l'action collective}

Paris, L'Harmattan, $465 \mathrm{p}$.

V oilà un ouvrage qui ne laissera pas indifférent.e.s celles et ceux qui mènent une réflexion sur les fondements, les principes et les stratégies de I'action collective à l'échelle des territoires et/ou qui sont impliqué.e.s dans les processus de développement, conseil ou animation à l'échelle territoriale. Couplant érudition et engagement dans l'action, Jean-Marie Funel affiche d'emblée le dessein de son essai dans un avant-propos qui résonne comme un plaidoyer pour une pensée systémique et complexe des mécanismes du développement territorial. Pour l'auteur, il y a comme une nécessité à témoigner, à transmettre et à revendiquer le recours à des outils de conceptualisation et d'action qui intègrent l'incertitude et la contingence des situations.

Jean-Marie Funel choisit de nourrir son propos de ses nombreuses expériences professionnelles et de ses lectures de grands penseurs français du $X X^{e}$ siècle. Agro-économiste de formation, initialement empreint des idéologies tiersmondistes des années 1970, Jean-Marie Funel a connu un parcours marqué par une succession d'illusions puis de désillusions, d'abord sur les terres africaines, notamment au Tchad puis au Niger où il est impliqué dans des projets de développement, ensuite en France, alors que les lois de décentralisation de G. Deferre dotent les collectivités territoriales de nouvelles compétences. Le livre est ainsi émaillé d'une vingtaine de cas concrets qui illustrent ou étayent les propos de I'auteur. L'ambition conceptuelle et méthodologique de J.-M. Funel s'ancre également dans le réexamen de penseurs en sciences sociales, philosophes, sociologues et anthropologues : les écrits d'E. Morin, M. Crozier et E. Friedberg, C. Lévi-Strauss ou encore A. Touraine sont ainsi convoqués tout au long de ce manifeste pour une vision systémique et complexe de l'acteur éclairé au sein de son système organisé.

Prévenons d'emblée les lectrices et les lecteurs: ici, aucune référence anglosaxonne et seule une dizaine de références bibliographiques sur les 62 citées datent des 5 dernières années. Par conséquent, n'attendez pas un positionnement par rapport aux controverses scientifiques en cours sur la société liquide de Z. Bauman ou encore les systèmes socio-écologiques d'E. Ostrom. N'allez pas rechercher non plus dans cet essai une discussion sur capital social, empowerment, place-based analyses ou encore capabilités des acteurs. L'ambition de J.-M. Funel, dans cet essai, est ailleurs : en capitalisant de façon pragmatique et réaliste les enseignements de ses propres expériences, il cherche à revisiter les références intellectuelles qui leur donnent sens pour contribuer à une action collective locale structurante. Nous pouvons certes déplorer parfois le recours au jargon sur le « processus systémique discursif fortement contingent ». Mais l'auteur prend également le soin d'appuyer sa réflexion sur de nombreux cas concrets et propose des orientations pour éviter les écueils d'approches " mécanistes, techniciennes et administrées ". II s'agit ainsi d'éviter le refuge dans des certitudes établies qui occulteraient la singularité des situations. À l'instar de l'embeddedness de M. Granovetter ou encore du caractère situé des processus économiques prônés par R. Salais et M. Storper, J.-M. Funel s'attache à resituer les enjeux locaux dans leur contexte englobant (le " système général ").

Pour autant, il ne s'agit pas non plus de promouvoir des démarches qui conduisent à une tétanisation des actions face à la profonde complexité des systèmes d'action. J.-M. Funel défend l'idée que des cadres d'action opérationnels 
existent et qu'avec des points de vigilance et des garde-fous (intégrer la diversité des réseaux d'action, identifier précisément les nœuds d'interrelations qui s'y nouent et des variables qui composent les systèmes étudiés, veiller à une collecte précise de données dans son contexte social...), il est possible de déployer des stratégies collectives qui répondent de façon pertinente aux enjeux posés initialement. Pour ce faire, I'acteur doit se forger une représentation, certes partielle du système englobant dans lequel il se situe (" allumer une infime partie locale du système général ») mais qui lui permet de réduire l'incertitude pour passer à l'action à partir de l'acquisition de savoirs et de connaissances stabilisées sur cette portion "éclairée " de la réalité.

Pour présenter et développer sa posture intellectuelle et les éléments de sa démarche d'analyse compréhensive, l'auteur a opté pour une structuration de son livre en quatre parties qui traitent successivement des principes de l'action locale, du territoire, des différents systèmes localisés et enfin, plus précisément, des systèmes d'action. J.-M. Funel déploie ainsi, au fil des pages, une analyse critique de certaines notions (" projet » qui conduirait à légitimer des modalités d'action linéaires et réductrices aux dépens des connaissances spécifiques à acquérir dans des contextes incertains, "territoire " auquel l'auteur préfère la notion de systèmes d'action...) et la présentation d'instruments concrets, éprouvés au cours de travaux et études dans lesquels l'auteur a été impliqué (à l'échelle de pays, communautés de communes, agglomérations, campus urbains...). Sont ainsi décrits avec précision des méthodes, démarches et outils visant à produire des connaissances depuis "l'intérieur " de façon à appréhender le plus finement possible la complexité des systèmes élémentaires en jeu: constitution de groupes de travail pour la production collaborative de données, inventaire des données à collecter pour mener une analyse structurelle, représentation graphique des interactions entre les variables conduisant à la construction de graphes de motricité-dépendance, etc.

Je ne restituerai pas le détail de ces quatre parties qui peuvent être lues comme un cheminement intellectuel conduisant (i) à définir les composantes d'un nouveau paradigme du changement structurel qui s'affranchisse d'une "vision corsetée du territoire » et (ii) à promouvoir des démarches systémiques d'action collective. Je pointerai plutôt deux principaux aspects qui illustrent, de mon point de vue, l'intérêt du raisonnement conduit par J.-M. Funel.

Le premier point que je mettrai en exergue est la volonté de réhabiliter les formes de régulation au cœur des processus de l'action collective. Partant du constat que, indépendamment des stratégies individuelles, le jeu collectif résulte de l'influence d'un système général qui édicte ses règles, l'auteur défend l'idée d'une analyse structurelle qui mette à jour les mécanismes de régulation qui sous-tendent les faits observés dans ce système donné. Celuici serait le garant de l'intégration des acteurs de par son rôle de régulateur au sein du jeu qui s'opère au travers des règles formelles ou informelles mais aussi de gestion des conflits et des différentes formes de négociations. Dans ce contexte, on perçoit l'importance, pour les acteurs, de connaître et de maîtriser les règles du jeu pour une implication et une prise de responsabilités dans les processus en cours. L'apprentissage collectif de ces formes de régulation devient dès lors un enjeu essentiel pour l'action locale. L'appropriation des connaissances est primordiale et passe, en particulier, par des méthodes de diagnostic territorial qui ne reposent pas sur une compilation de simples monographies ou la seule collecte de données externes mais qui soient le produit des parties prenantes du territoire étudié. On se situe là au cœur de processus collaboratifs 
où la démarche d'investigation est coconstruite. Ce jeu collectif va toutefois prendre des formes différentes selon le type de système de pensée dans lequel évolue l'acteur.

Le second point que je souhaitais relever porte précisément sur l'identification de deux principaux modes de pensée dont on pourrait aisément déduire des formes distinctes d'ingénierie territoriale ou d'accompagnement des processus de développement local. En s'inspirant des écrits de C. Lévi-Strauss, J.-M. Funel distingue deux modes de pensée. Le premier relève de la pensée thématique qui serait l'apanage de l'ingénieur privilégiant un raisonnement à base de théories et de formulation d'hypothèses, d'optimisation de moyens en vue de produire des résultats. Comment ne pas penser, à ce propos, aux injonctions du New Public Management et le recours aux démarches de rationalisation des choix budgétaires et de recherche d'efficacité et d'efficience ? Le second mode de pensée est celui de la pensée sociétale qui caractériserait le bricoleur, ce dernier misant sur son intuition et son imagination pour agir dans un environnement donné dont il connaît les spécificités. "Le bricoleur s'arrange avec les moyens du bord, c'est-à-dire avec un ensemble fini d'outils et de matériaux hétéroclites pour arriver à un résultat dont la composition est la conséquence d'occasions et d'opportunités plutôt que d'un projet. " Selon la logique du bricoleur, on passerait ainsi d'une gestion du développement local non pas par appel à projets, mais par "appels à collaborations " gérés par les groupes d'action locale et non par les institutions, dans des modèles d'action collective co-construits et collaboratifs.

Je recommande donc cette lecture intellectuellement stimulante à toutes les personnes, étudiant.e.s, enseignant.e.s, responsables associatifs ou cadres dans une organisation professionnelle agricole, qui s'interrogent sur les modalités $d$ 'intervention collective à l'échelle des territoires et cherchent à (re)définir leurs stratégies d'action. Cet essai, véritable agitateur de neurones et stimulateur de curiosités, invite ainsi à sortir des sentiers battus des politiques de projet trop formatées et à s'éloigner de modes de pensée certes efficaces mais souvent réducteurs, simplificateurs et court-termistes. Ce livre peut enfin éclairer les acteurs du développement des territoires qui ne recherchent pas de solution "clé en main " mais misent davantage sur des démarches collaboratives singulières du type living lab, à l'instar de ce qui est fait dans le cadre de l'expérimentation, citée dans ce livre, sur les territoires zéro chômeur de longue durée. Le développement des démarches d'analyse structurelle telles que celle proposée dans cet ouvrage ouvre ainsi des pistes prometteuses pour permettre aux acteurs de disposer de connaissances partagées sur leurs lieux de vie et de travail et sur les règles de régulation du système dans lequel ils évoluent. Une telle perspective apparaît comme une condition pour retenir localement des choix stratégiques pertinents et durables.

Marielle BERRIET-SOLLIEC Professeure d'économie à AgroSupDijon 